\title{
Pseudopterosin synthesis from a chiral cross-conjugated hydrocarbon through a series of cycloadditions
}

\author{
Christopher G. Newton', Samuel L. Drew', Andrew L. Lawrence ${ }^{11}$, Anthony C. Willis', \\ Michael N. Paddon-Row ${ }^{2 \star}$ and Michael S. Sherburn'^
}

\begin{abstract}
The pseudopterosins are a family of diterpene marine natural products, which, by virtue of their interesting antiinflammatory and analgesic properties, have attracted the attentions of many synthetic chemists. The most efficient syntheses reported to date are 14 and 20 steps in the longest linear sequence for chiral pool and enantioselective approaches, respectively, and all start with precursors that are easily mapped onto the natural product structure. Here, we describe an unconventional approach in which a chiral cross-conjugated hydrocarbon is used as the starting material for a series of three cycloadditions. Our approach has led to a significant reduction in the step count required to access these interesting natural products (10 steps chiral pool and 11 steps enantioselective). Furthermore it demonstrates that crossconjugated hydrocarbons, erroneously considered by many to be too unstable and difficult to handle, are viable precursors for natural product synthesis.
\end{abstract}

\footnotetext{
T
} he pseudopterosins (Table 1) comprise the largest family of amphilectane diterpenes, with 31 members isolated to date, all of which are derived from one of three stereoisomeric aglycones $^{1}$. The remaining structural diversity arises from the nature of the sugar, the site of glycosylation and the extent of sugar acetylation. Members of the pseudopterosin family have been shown to exhibit a wide range of biological activities, including anticancer ${ }^{2}$, antimalarial $^{2}$ and (perhaps most notably) anti-inflammatory properties that exceed the potencies of existing drugs such as indomethacin ${ }^{3,4}$.

The pseudopterosins have been the focus of a large volume of synthetic work over the past 25 years due to their challenging structures and pronounced biological activities. To date, 14 total and formal syntheses of pseudopterosin aglycones have been published $(\text { Table } 1)^{5-18}$. This substantial archive of outstanding synthetic contributions pinpoints the pseudopterosin family as an ideal vehicle for the development of a progressively original chemical synthesis. All previous syntheses of these chiral tricyclic hexahydro-phenalenes deploy either chiral monoterpenes or substituted benzenes as starting materials, which are converted into pseudopterosins through sequences of chain extensions and annulations. These earlier approaches are examples of 'structure-goal' strategies ${ }^{19}$. Specifically, a commercially available terpene or aromatic precursor is identified that maps onto a section of the pseudopterosin target structure. Herein, we disclose the successful synthetic realization of a 'transform-based' strategy ${ }^{19}$ to a pseudopterosin, an approach that does not commence with a 'mappable' commercial precursor and instead employs a powerful, triple cycloaddition sequence of a highly reactive cross-conjugated precursor to generate the natural product framework in very short order.

\section{Results and discussion}

Our retrosynthetic analysis is presented in Fig. 1. Viewing the catechol A-ring of pseudopterosin (-)-G-J aglycone as its 1,2-diketone tautomer 1 unveils the possibility of a Diels-Alder (DA) disconnection back to conjugated diene 2 and ethylene dione 3 as dienophile. The cyclohexene B-ring of diene 2 can be disconnected further, through a second DA transform, to provide cyclic [3] dendralene 4 and ethylene as a dienophile. A final DA disconnection of the cyclohexene C-ring of cyclic [3] dendralene 4 reveals substituted 1,1-divinylallene 6 , together with 4-methyl-1,3-pentadiene 5 as dienophile.

Thus, the tricyclic framework of the natural product is exploded into four acyclic precursors through the consecutive disconnection of three pairs of covalent bonds. In the synthetic direction, issues of chemoselectivity, regioselectivity and stereoselectivity in each of the three cycloadditions would need to be overcome, in addition to the potentially problematic preparation and handling of crossconjugated hydrocarbon 6 . The presence of both $E$ - and Z-configured propenyl-substituents in substituted divinylallene 6 confers axial chirality upon the structure, and hence the possibility of a substrate-controlled stereoselective synthesis.

The preparation of chiral 1,1-divinylallene 6 in enantiomerically enriched form represented the first significant challenge of this synthesis. Our recent successful preparation of the parent 1,1-divinylallene revealed the hydrocarbon's susceptibility to DA polymerization, a characteristic that, in combination with the low boiling point of the substance, dictated a somewhat lengthy synthesis ${ }^{20}$. We ventured that chiral trimethyl analogue 6 would be both less volatile and less prone to self-immolation than its parent, and consequently set about its synthesis in a significantly more direct manner (Fig. 2). Thus, homologation of crotonaldehyde 7 into the terminal alkyne under Colvin-Hamill conditions ${ }^{21-23}$, then deprotonation and trapping with the Weinreb amide of acetic acid, furnished ketone 9 in $62 \%$ yield in one pot, thereby avoiding the need to isolate the low boiling pentenyne intermediate 8 . Catalytic enantioselective reduction of the ketone function of 9 under Noyori conditions $^{24}$ gave propargylic secondary alcohol 10 in high

${ }^{1}$ Research School of Chemistry, Australian National University, Canberra, ACT 2601, Australia, ${ }^{2}$ School of Chemistry, The University of New South Wales, Sydney, New South Wales 2052, Australia; †resent address: School of Chemistry, University of Edinburgh, Joseph Black Building, West Mains Road, Edinburgh EH9 3JJ, UK. *e-mail: michael.sherburn@anu.edu.au; m.paddonrow@unsw.edu.au 
Table 1 | Previous starting material goal-based approaches to the pseudopterosins and the present transform-based strategy.

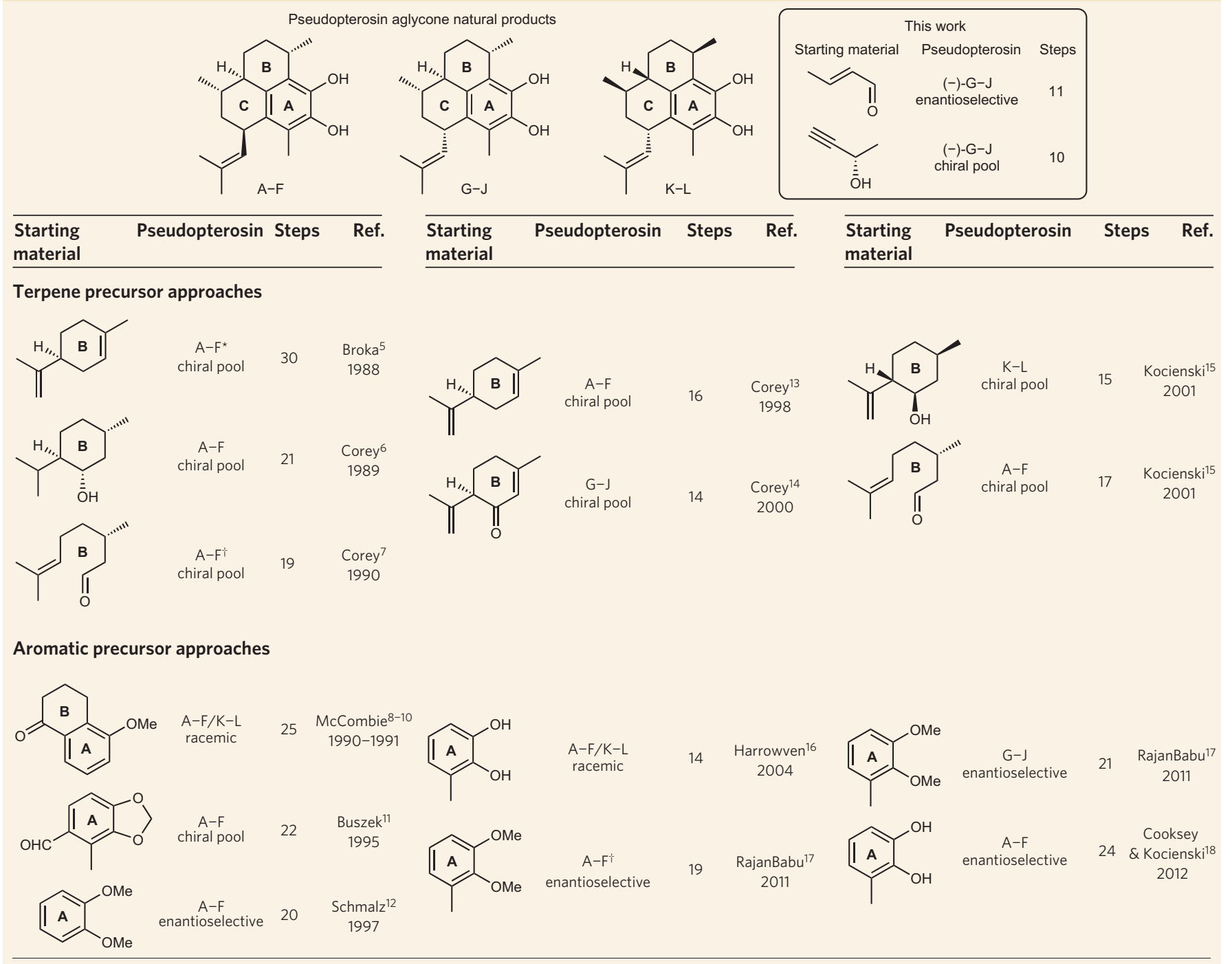

${ }^{\star}$ Synthesis of a protected derivative. ${ }^{\dagger}$ Formal synthesis. Step counts are reported as the longest linear sequence.

enantiopurity. This compound could also be synthesized in one step from the commercially available and highly enantioenriched propargylic alcohol 11. Thus, Sonogashira cross-coupling between (S)-3-butyn-2-ol 11 and (E)-1-bromo-1-propene 12 worked extremely well, furnishing enyne 10 in $94 \%$ yield. Alcohol 10, accessed either through two-step enantioselective synthesis or through the one-step 'chiral pool' pathway, was converted into the corresponding methanesulfonate derivative 13 as a prelude to the critical $\mathrm{C}-\mathrm{C}$ bond-forming step, which would result in the preparation of chiral cross-conjugated hydrocarbon 6 and a switch from a substance with point chirality into one with axial chirality.

After extensive experimentation, we unearthed conditions to generate hydrocarbon 6 by cross-coupling electrophile 13 with Grignard reagent 14 . Our Ni(0)-catalysed Kumada cross-coupling proceeded with a high level of formal anti- $S_{N} 2^{\prime}$ selectivity, thereby furnishing a highly enantiomerically enriched product and, moreover, one that can be readily produced on multigram scale. The absolute configuration of hydrocarbon 6 was deduced through its conversion into more stable derivatives (vide infra).

The DA cycloaddition is one of the most successful reactions in total synthesis ${ }^{25}$. Nevertheless, the novelty of all three contexts proposed in this synthesis caused us to be apprehensive. Of the three, we were particularly concerned about the one involving hydrocarbon 6, due to the dearth of reported examples involving axially chiral vinylallenes as dienes. We therefore modelled this process computationally using the B3LYP level of theory (see Supplementary Section 'Computational' for details). Scouting experiments in the laboratory uncovered the need to both replace the isobutenyl-substituent of dienophile 5 (Fig. 1) with an ester function and to include a formyl activating group at the other dienophilic carbon. Of the 43 transition structures (TSs) located for the DA addition of $E$-(carboxymethyl)acrolein 15-Me to the 1-Emethylbutadiene component of 6 , the lowest-energy TS, TS-1, predicted the formation of cycloadduct $\mathbf{1 6}$ and set the scene for the successful completion of the total synthesis (Fig. 3).

Control of both orientational regioselectivity and stereoselectivity in the first cycloaddition $(6 \rightarrow 16)$ were needed. TS-1 (Fig. 3) displays significant bond-forming asynchronicity, with forming bond lengths of 2.131 and $2.661 \AA(\Delta r=0.53 \AA)$. This asynchronicity confers a degree of biradicaloid character to TS-1 and this is best stabilized by making the forming bond involving the allenic carbon the shorter of the two, thereby conferring pentadienyl radicaloid character to the divinylallene. The dienophile component acquires radicaloid character at the longer bond-forming carbon centre and, because the formyl group is a more potent radical stabilizer than the methoxycarbonyl group, the observed 

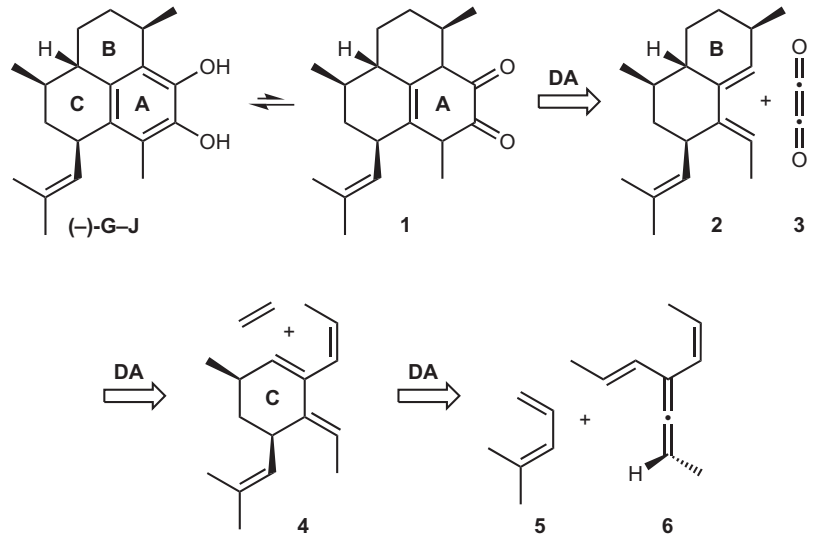

Figure 1 | Strategic bond disconnections pursued in this study.

Retrosynthetic analysis of the pseudopterosin (-)-G-J aglycone reveals the triple DA disconnection to axially chiral 1,1-divinylallene 6 .

orientational preference follows. Our calculations reveal the TS with the opposite orientation to TS-1 lies $4.9 \mathrm{~kJ} \mathrm{~mol}^{-1}$ higher in energy. The endo- $\mathrm{CO}_{2} \mathrm{Me}$ mode of dienophile addition is favoured over the alternative exo mode by $1.1 \mathrm{~kJ} \mathrm{~mol}^{-1}$, and the allenic methyl group's preference for anti over syn is $8.5 \mathrm{~kJ} \mathrm{~mol}^{-1}$. Both preferences, together with the finding that the latter is stronger than the former, may be understood by noting that the combination of the forming bond at the allenic centre and the allenic $\mathrm{C}=\mathrm{C}-\mathrm{H}$ group in TS-1 and the other TSs form a quasi-allylic system with an exo-H-C1-C2-C3 dihedral angle of $12^{\circ}$ (as compared with $0^{\circ}$ in propene) and a C1-C3 distance of $2.86 \AA$ (as compared with $2.51 \AA$ in propene). This quasi-allylic unit should thus be sensitive to the presence of 1,3-allylic strain, which explains the allenic methyl group's anti preference and the favoured endo- $\mathrm{CO}_{2} \mathrm{Me}$ disposition in TS-1.

In the laboratory, the optimized first DA reaction was carried out on decagram scale and, most conveniently, in tandem with the synthesis of hydrocarbon 6. Thus, when the Kumada cross-coupling reaction was deemed complete, excess Grignard reagent 14 was quenched by the addition of methanol, then commercially available dienophile 15-Et was injected into the reaction flask. The one-pot cross-coupling/DA sequence delivered adduct 16 in $61 \%$ overall yield (d.r. $=5: 1: 1)$ from alcohol 10 (a distinctly lower yield was obtained by conducting this sequence in two separate flasks) while maintaining a high level of enantiopurity over the point-toaxial-to-point chirality transfer.

The clean conversion of hydrocarbon 6 into DA adduct 16 not only reflects the unusually high reactivity of 1,1-divinylallenes as $4 \pi$ cycloaddition partners, but also the low reactivity of the $s$-cis diene component of $\mathbf{1 6}$ towards further reaction. Indeed, the lack of reactivity of the 1,3-butadiene component of 16 , coupled with its similar reactivity to the diene group of adduct 17 , almost undermined the synthesis. This problem was ultimately solved by halting the high-pressure reaction between triene 16 and the chosen dienophile, acrolein, at low levels of conversion, thereby minimizing the amount of unwanted acrolein double cycloaddition product. Unreacted precursor was easily re-isolated and recycled, thereby furnishing an acceptable overall yield of product 17 . This reaction exhibits high regio- and stereoselectivity, with the acrolein dienophile approaching the diene from the face opposite to that in which the C3-methyl group resides. The two-now superfluous-dienophile activating groups were removed by deformylation with Wilkinson's complex ${ }^{26}$. Ester $\mathbf{1 8}$ was then subjected to a one-pot selective reduction/olefination sequence to provide hydrocarbon 19 in 75\% yield, thus setting the scene for the final cycloaddition.

Both strategically and conceptually, the third cycloaddition is perhaps the most interesting of the three. To our knowledge, catechol synthesis by way of a DA reaction has not been reported before. Since ethylene dione 3 (Fig. 1) has a fleeting existence under normal working conditions ${ }^{27}$, a synthetic equivalent was required. Following extensive testing involving several potential candidates, we ultimately elected to employ a synthetic equivalent of ketene and introduce the second ketone through oxidation. Thus, following a cycloaddition between hydrocarbon 19 and nitroethylene at $19 \mathrm{kbar}$ and ambient temperature to give tricycle 20, a Nef reaction gave ketone $21^{28}$. Kinetic enolate formation and electrophilic oxygenation with Davis' oxaziridine gave the resulting a-hydroxy ketone 22, which was oxidized to the pseudopterosin (-)-G-J aglycone under Swern conditions. Analytical chiral HPLC analysis of synthetic pseudopterosin G-J aglycone prepared in this manner against an authentic natural sample allowed for the assignment of absolute configuration as the (-)-G-J enantiomer (see Supplementary Fig. 3 for details).

The synthesis described here should be readily amenable to the preparation of the two other pseudopterosin aglycones. Thus, epimerization of ester $\mathbf{1 8}$ followed by a repeat of the same five-step sequence shown in Fig. 3 will allow the formation of pseudopterosin $\mathrm{K}-\mathrm{L}$ aglycone (Table 1). Pseudopterosin A-F aglycone (Table 1) will then be accessible by simply employing either the enantiomeric Noyori catalyst or the enantiomer of the chiral pool precursor employed in this study (Fig. 2).

In summary, the pursuit of a transform-based strategy has culminated in the shortest catalytic enantioselective (11 steps) and chiral pool (10 steps) total syntheses of a pseudopterosin natural product. The synthesis constructs all three rings of the tricyclic natural product via a triple DA reaction sequence commencing with an

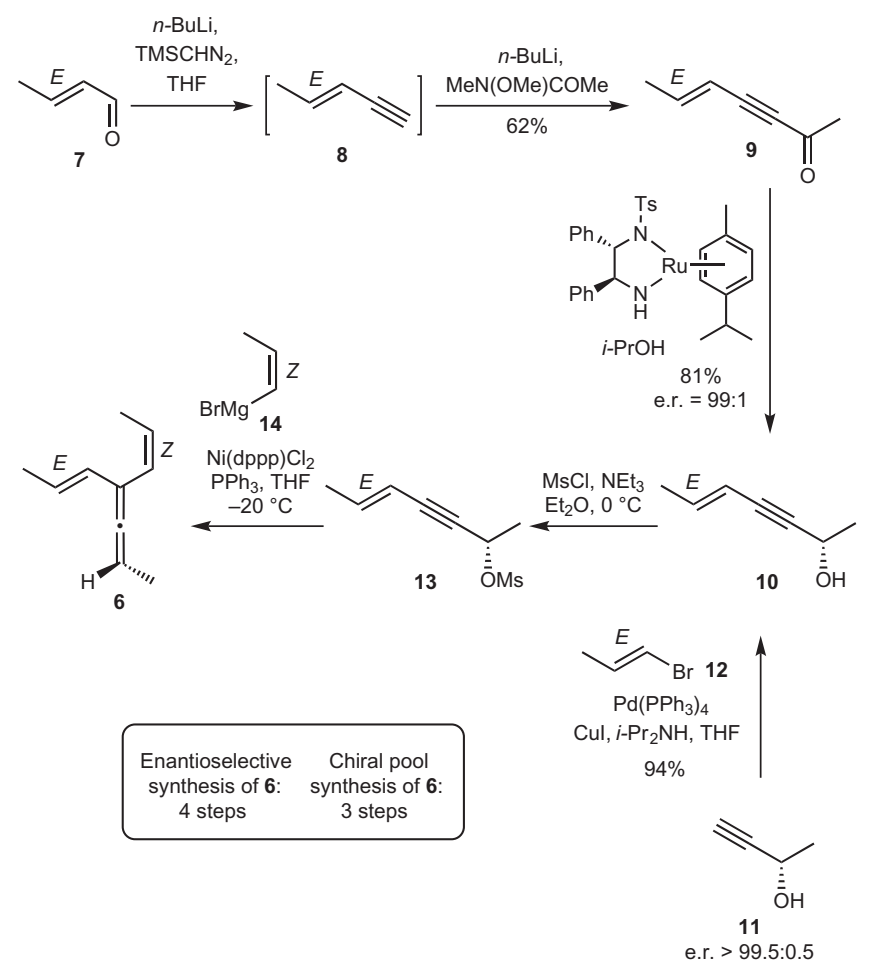

Figure 2 | Two synthetic approaches to enantioenriched substituted 1,1-divinylallene 6. Enantioenriched propargylic alcohol 10 was prepared in two steps from crotonaldehyde 7 via a one-pot homologation/substitution sequence, followed by an enantioselective reduction under Noyori conditions. Alternatively, this intermediate could be accessed in one step from commercially available chiral pool alcohol 11 . Conversion to mesylate 13 allowed for the key Kumada cross-coupling reaction to provide allene 6, thus permitting exploration of its DA reactivity. 


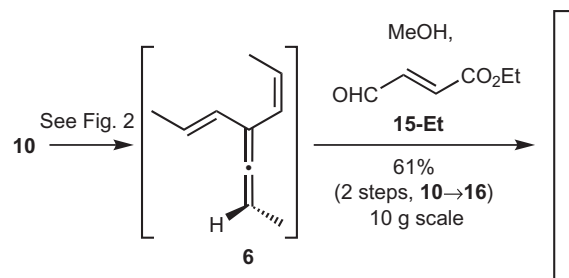
Lowest energy B3LYP transition structure
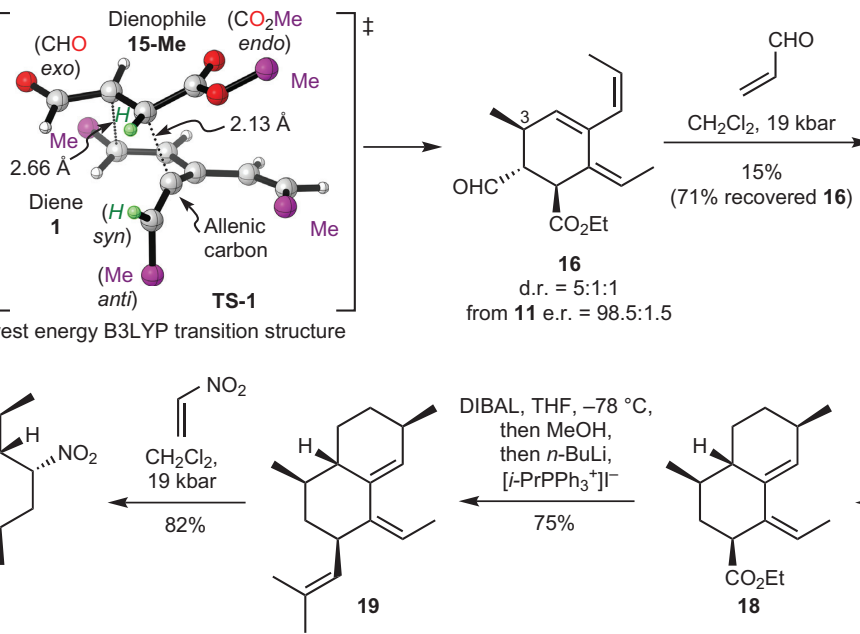
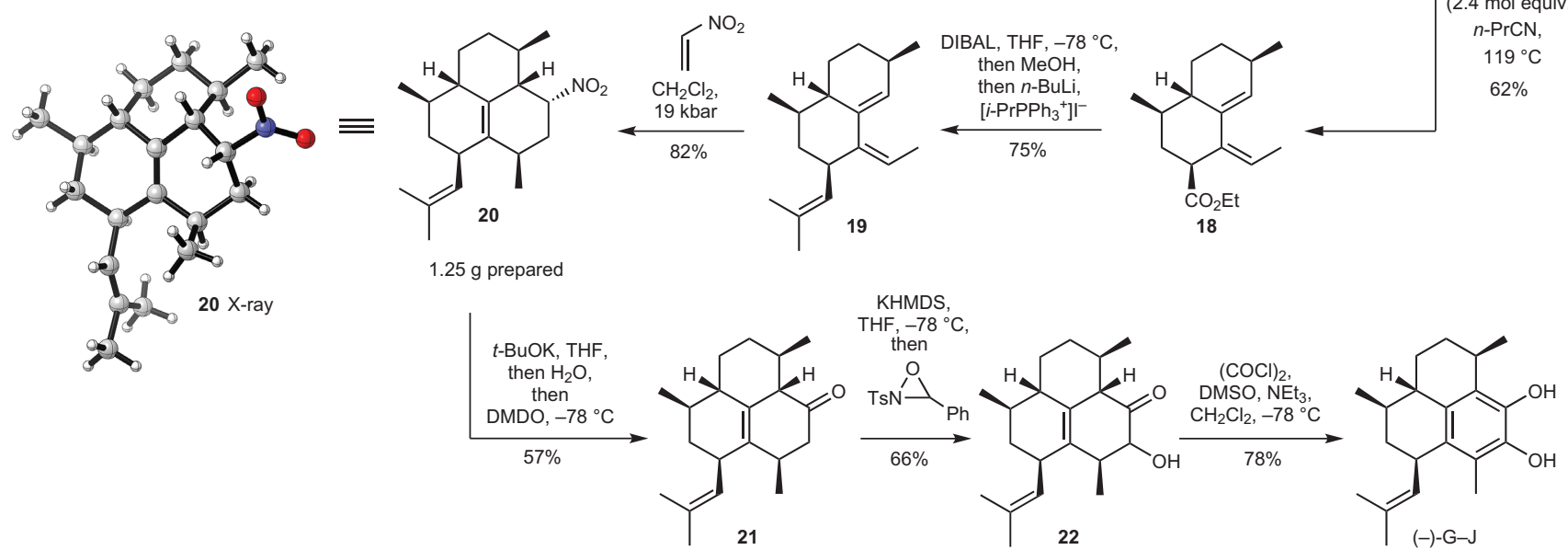

Figure 3 | Total synthesis of pseudopterosin (-)-G-J aglycone employing a diene-transmissive triple DA cycloaddition strategy. Generation of substituted 1,1-divinylallene 6 in two steps from alcohol 10, followed by an in situ DA reaction with dienophile 15-Et, gave cyclic cross-conjugated hydrocarbon 16. Throughout this transformation, enantiopurity is retained during a point-to-axial-to-point chirality transfer and, as predicted by density functional theory (DFT) calculations (lowest-energy TS structure TS-1), formation of the desired diastereomer 16 was favoured. The second DA reaction, this time with acrolein as dienophile, provided dialdehyde 17, which was subjected to a double decarbonylation followed by a one-pot reduction/olefination sequence to afford hydrocarbon 19. A final cycloaddition employing nitroethylene as a ketene equivalent completed formation of the carbon skeleton. The final stage of the synthesis involved a Nef reaction/ketone $\alpha$-hydroxylation/Swern oxidation sequence, generating the pseudopterosin (-)-G-J aglycone in 11 steps (enantioselective) or 10 steps (chiral pool).

axially chiral, substituted 1,1-divinylallene. Novel and notable features of this highly unorthodox approach, which will find wider application, include (1) a new variation on the cross-coupling theme to prepare hydrocarbon 6 ; (2) the stereoselective cycloaddition of axially chiral divinylallene 6 ; (3) a point-to-axial-topoint chirality manoeuver with retention of enantiopurity; and (4) a novel DA reaction-based catechol synthesis. This work is perhaps the most extreme incarnation yet of the potency of the DA reaction in natural product synthesis, and one that signals the coming of age of cross-conjugated hydrocarbons in this domain.

Received 29 May 2014; accepted 9 October 2014; published online 17 November 2014

\section{References}

1. Berrué, F., McCulloch, M. W. B. \& Kerr, R. G. Marine diterpene glycosides. Bioorg. Med. Chem. 19, 6702-6719 (2011).

2. Rodríguez, I. I. et al. New pseudopterosin and seco-pseudopterosin diterpene glycosides from two Colombian isolates of Pseudopterogorgia elisabethae and their diverse biological activities. J. Nat. Prod. 67, 1672-1680 (2004).

3. Look, S. A., Fenical, W., Jacobs, R. S. \& Clardy, J. The pseudopterosins: antiinflammatory and analgesic natural products from the sea whip Pseudopterogorgia elisabethae. Proc. Natl Acad. Sci. USA 83, 6238-6240 (1986).

4. Look, S. A., Fenical, W., Matsumoto, G. K. \& Clardy, J. The pseudopterosins: a new class of anti-inflammatory and analgesic diterpene pentosides from the marine sea whip Pseudopterogorgia elisabethae (Octocorallia). J. Org. Chem. 51, 5140-5145 (1986).

5. Broka, C. A., Chan, S. \& Peterson, B. Total synthesis of (-)-pseudopterosin A. J. Org. Chem. 53, 1584-1586 (1988).

6. Corey, E. J. \& Carpino, P. Enantiospecific total synthesis of pseudopterosins A and E. J. Am. Chem. Soc. 111, 5472-5474 (1989).

7. Corey, E. J. \& Carpino, P. A new enantiospecific route to the pseudopterosins. Tetrahedron Lett. 31, 3857-3858 (1990).
8. Ganguly, A. K., McCombie, S. W., Cox, B., Lin, S. \& McPhail, A. T. Stereospecific synthesis of the aglycone of pseudopterosin E. Pure Appl. Chem. 62, 1289-1291 (1990).

9. McCombie, S. W., Cox, B., Lin, S-I., Ganguly, A. K. \& McPhail, A. T. Controlling benzylic functionality and stereochemistry: 1 . Synthesis of the secopseudopterosin aglycone. Tetrahedron Lett. 32, 2083-2086 (1991).

10. McCombie, S. W., Cox, B. \& Ganguly, A. K. Controlling benzylic functionality and stereochemistry: 2. Synthesis of the pseudopterosin aglycone. Tetrahedron Lett. 32, 2087-2090 (1991).

11. Buszek, K. R. \& Bixby, D. L. Total synthesis of pseudopterosin A and E aglycon. Tetrahedron Lett. 36, 9129-9132 (1995).

12. Majdalani, A. \& Schmalz, H-G. Enantioselective synthesis of the aglycones of pseudopterosin and seco-pseudopterosin via a common synthetic intermediate. Synlett 1303-1305 (1997).

13. Corey, E. J. \& Lazerwith, S. E. A direct and efficient stereocontrolled synthetic route to the pseudopterosins, potent marine anti-inflammatory agents. J. Am. Chem. Soc. 120, 12777-12782 (1998).

14. Lazerwith, S. E., Johnson, T. W. \& Corey, E. J. Syntheses and stereochemical revision of pseudopterosin G-J aglycon and helioporin E. Org. Lett. 2, 2389-2392 (2000).

15. Kocienski, P. J., Pontiroli, A. \& Qun, L. Enantiospecific syntheses of pseudopterosin aglycones. Part 2. Synthesis of pseudopterosin K-L aglycone and pseudopterosin A-F aglycone via a B $\rightarrow$ BA $\rightarrow$ BAC annulation strategy. J. Chem. Soc. Perkin Trans 1 2356-2366 (2001).

16. Harrowven, D. C. \& Tyte, M. J. Total synthesis of ( \pm )-pseudopterosin A-F and K-L aglycone. Tetrahedron Lett. 45, 2089-2091 (2004).

17. Mans, D. J., Cox, G. A. \& RajanBabu, T. V. Ethylene in organic synthesis. Repetitive hydrovinylation of alkenes for highly enantioselective syntheses of pseudopterosins. J. Am. Chem. Soc. 133, 5776-5779 (2011).

18. Cooksey, J. P., Kocieński, P. J., Schmidt, A. W., Snaddon, T. N. \& Kilner, C. A. A synthesis of the pseudopterosin A-F aglycone. Synthesis 44, 2779-2785 (2012).

19. Corey, E. J. \& Cheng, X-M. The Logic of Chemical Synthesis (Wiley, 1995).

20. Cergol, K. M. et al. 1,1-Divinylallene. Angew. Chem. Int. Ed. 50, 10425-10428 (2011). 
21. Colvin, E. W. \& Hamill, B. J. One-step conversion of carbonyl compounds into acetylenes. J. Chem. Soc. Chem. Commun. 151-152 (1973).

22. Colvin, E. W. \& Hamill, B. J. A simple procedure for the elaboration of carbonyl compounds into homologous alkynes. J. Chem. Soc. Perkin Trans. 1 869-874 (1977).

23. Hansen, E. C. \& Lee, D. Efficient and Z-selective cross-metathesis of conjugated enynes. Org. Lett. 6, 2035-2038 (2004).

24. Matsumura, K., Hashiguchi, S., Ikariya, T. \& Noyori, R. Asymmetric transfer hydrogenation of $\alpha, \beta$-acetylenic ketones. J. Am. Chem. Soc. 119, 8738-8739 (1997).

25. Nicolaou, K. C., Snyder, S. A., Montagnon, T. \& Vassilikogiannakis, G. The Diels-Alder reaction in total synthesis. Angew. Chem. Int. Ed. 41, 1668-1698 (2002).

26. Tsuji, J. \& Ohno, K. Organic syntheses by means of noble metal compounds XXI. Decarbonylation of aldehydes using rhodium complex. Tetrahedron Lett. 6, 3969-3971 (1965).

27. Schröder, D. et al. Ethylenedione: an intrinsically short-lived molecule. Chem. Eur. J. 4, 2550-2557 (1998).

28. Adam, W., Mąkosza, M., Saha-Möller, C. R. \& Zhao, C-G. A mild and efficient Nef reaction for the conversion of nitro to carbonyl group by dimethyldioxirane (DMD) oxidation of nitronate anions. Synlett 1335-1336 (1998).

\section{Acknowledgements}

The authors thank R. Kerr and F. Berrué for providing authentic samples of the pseudopterosins, H-G. Schmalz for providing a copy of the PhD thesis of A. Majdalani, S. M. (M.) Tan and E. Lindeboom for preliminary experiments, and A. Herlt for assistance with HPLC. M.N.P-R. acknowledges that this research was undertaken with the assistance of resources provided at the NCI National Facility through the National Computational Merit Allocation Scheme supported by the Australian Government. This work was supported by the Australian Research Council.

\section{Author contributions}

C.G.N., S.L.D., A.L.L. and M.S.S. conceived, designed and carried out the synthetic experiments. A.C.W. performed the crystallographic studies. M.N.P-R. designed and carried out the computational study. All authors discussed and co-wrote the manuscript.

\section{Additional information}

Supplementary and chemical compound information are available in the online version of the paper. Reprints and permissions information is available online at www.nature.com/reprints. Correspondence and requests for materials should be addressed to M.N.P-R. (computational) or M.S.S. (synthetic).

\section{Competing financial interests}

The authors declare no competing financial interests. 